\title{
A Case of Split Cord Malformation with Triple Bony Spur in a Single Dural Sleeve-A Classification Dilemma
}

\author{
Saurabh Kumar Verma ${ }^{1}$ Madakasira Sitaram Sridhar ${ }^{1} \quad$ Manish Garg ${ }^{1}$ \\ ${ }^{1}$ Department of Neurosurgery, Army Hospital (Research \& Referral), \\ New Delhi, India \\ Address for correspondence Saurabh Kumar Verma, MCh, \\ Department of Neurosurgery, Army Hospital (Research \& Referral), \\ Dhaulakuan, Delhi Cantt, New Delhi 110010, India \\ (e-mail: eskay6362@yahoo.co.in).
}

Indian J Neurosurg 2018;7:275-277

\begin{abstract}
Keywords

- diastematomyelia

- bony spur

- split cord
\end{abstract}

\section{Introduction}

Split cord malformation (SCM) is an uncommon congenital anomaly in which a segment of the spinal cord is divided into two parts by a fibrous or rigid bony spur. The bony septum usually originates from the vertebral body and passes posteriorly, causing splitting of the cord.

The most widely accepted theory about the embryogenesis of these complex malformations was originally proposed by Bremer in $1952^{1}$ and subsequently modified by Pang et $\mathrm{al}^{2,3}$ as the "unified theory of embryogenesis." The basic error appears to be the development and persistence of "accessory neurenteric canal." According to the theory, these lesions were divided into two types depending on the type of the midline mesenchymal derivative and the dural investment of the hemicords. SCM-I is characterized by the presence of double dural sacs, with rigid extradural bony/cartilaginous spur leading to symmetrical or asymmetrical division of the cord. In SCM-II, there is a single dural sac with a nonrigid fibrous spur and symmetrical division of the cord. SCM-II are slightly more common than SCM-I, constituting around 50 to $60 \%$ of SCMs. ${ }^{2,3}$
The authors report a unique case of SCM with triple bony spurs lying both intra- and extradurally in a single dural sleeve.

At the time of submission of this report, to the best of authors' knowledge, no case of SCM with triple bony spurs in single dural sleeve has been reported anywhere in the world literature.

\section{Case Summary}

This was a case of a 3-year-old male child, who presented to the center with complaints of congenital deformity of spine and progressively worsening scoliosis of the spine along with abnormal hairy patch over midline in the back.

Clinically the patient had scoliosis to the right along with hypertrichosis. He, however, had no neurologic deficit and had no lower limb weakness or sphincter disturbance.

\section{Magnetic Resonance Imaging of Head was Normal} Magnetic resonance imaging (MRI) of the spine dated October 21, 2016 showed scoliosis in dorsal spine to right; received

December 26, 2017

accepted

May 18, 2018

published online

August 30, 2018
DOI https://doi.org/

10.1055/s-0038-1670019.

ISSN 2277-954X.

\section{(C)2018 Neurological Surgeons' \\ Society of India}

License terms

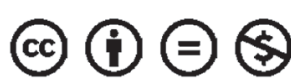


multiple segmentation anomalies in the dorsal, lumbar, and sacral levels; splitting of the cord from D4 to L2 level; and three bony spurs at D6, D10, and D12 levels ( - Fig. 1a, b) along with low-lying tethered cord with conus at the level of lower border of $\mathrm{L} 3$ vertebra along with a dorsal dermal sinus at sacral level. Computed tomographic (CT) of the spine showed bony spurs at the level of D6, D10, and D12 vertebrae (-Fig. 1c-f).

The patient was taken up for surgery and exploration. The site of split was explored first after adequately removing the spur and other tethering elements at the site. Filum de-tethering was performed in a single stage. Perioperatively the findings of MRI scan were confirmed in the form of there being a single dural sleeve ( $\mathbf{- F i g}$. 2a-c). The bony spurs were intradural for the maximum extent; however, because the bony spurs were attached to both the posterior aspect of the body of vertebrae and to the lamina after piercing out through the dural sleeve both ventrally and dorsally, they were technically deemed to be both intra- and extradural. The postoperative period remained uneventful, and at 4 months after surgery, he had no deficits.

\section{Discussion}

SCM-I is characterized by the presence of double dural sacs, with rigid extradural bony/cartilaginous spur leading into symmetrical or asymmetrical division of the cord. In SCM-II, there is a single dural sac with a nonrigid fibrous spur and symmetrical division of the cord. Among all the types of SCM, the composite-type SCM is very rare and results from two separate foci of ecto-endodermal adhesions and endomesenchymal tracts ${ }^{3}$ leading to development of different SCM types with intervening normal cord in the same patient. ${ }^{4}$ According to a classification system proposed by Mahapatra and Borkar, ${ }^{5}$ SCM-I has been subclassified as follows:

- Type Ia: Bony spur in the center with equally duplicated cord above and below the spur

- Type Ib: Bony spur at the superior pole with no space above and a large duplicated cord lower down
- Type Ic: Bony spur at the lower pole with a large duplicated cord above

- Type Id: Bony spur straddling the bifurcation with no space above or below the spur

Only a few cases of composite-type SCM have been reported in the literature.

In this report, the authors present a case of case of SCM with triple bony spur in a single dural sleeve and multiple associated bony anomalies.

This case does not fit into any of the previously mentioned classification system.

\section{Conclusion}

The authors report the first documented case of "SCM with triple bony spurs in a single dural sleeve." This case does not fit into any of the existing classification system of SCM

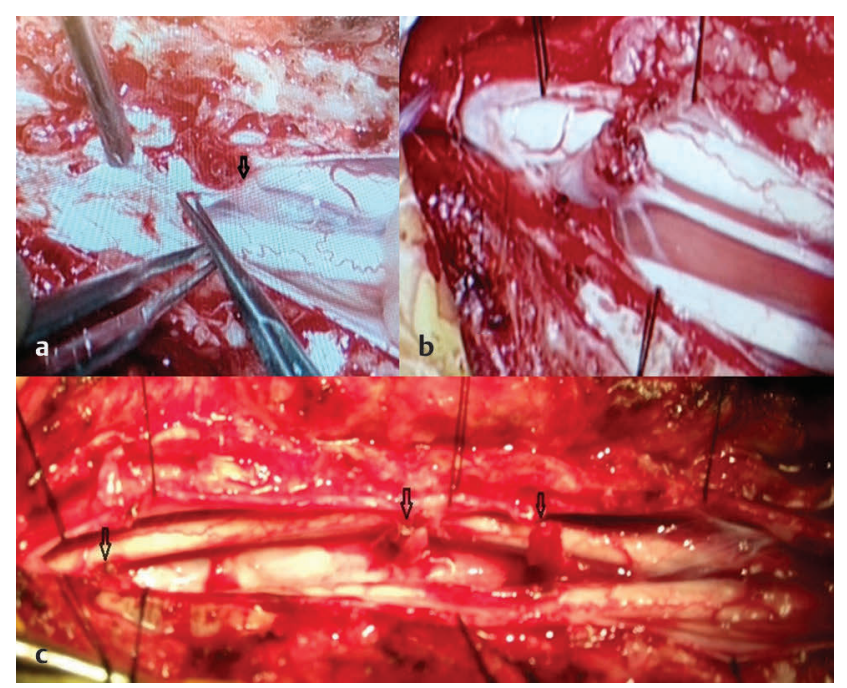

Fig. 2 (a) Intraoperative image showing single dural sleeve-one spur visible (arrow). (b) Intraoperative image showing single dural sleeve-one spur visible at another level. (c) Intraoperative image showing single dural sleeve-all three spurs (arrows) visible.

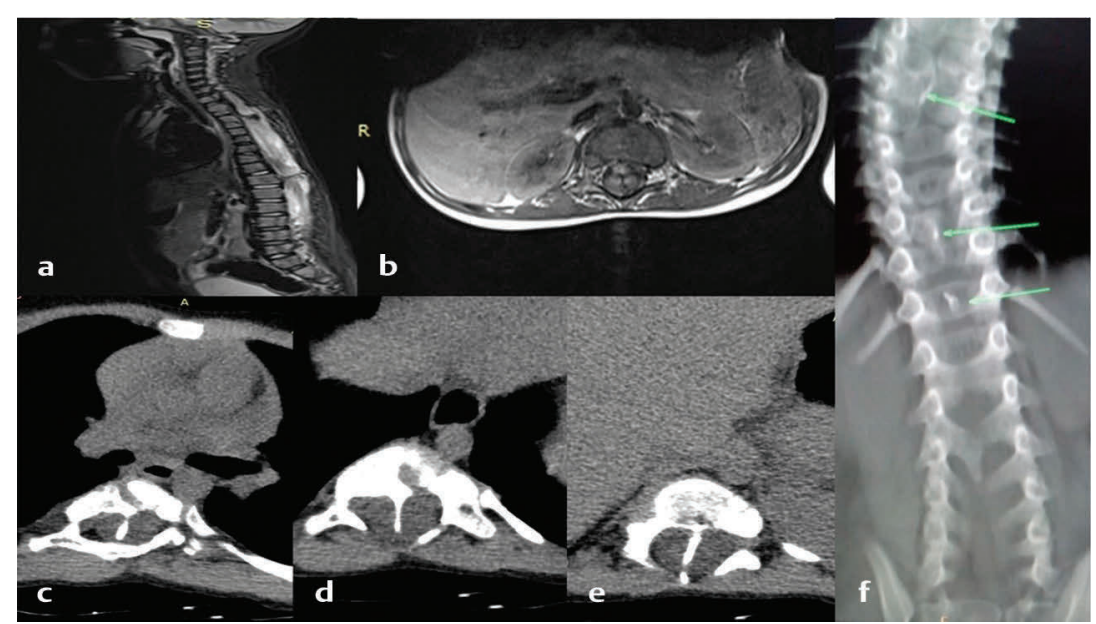

Fig. 1 (a) Sagittal section whole spine MRI image. (b) Axial section MRI of the spine showing bony spur. (c-e) Axial section NCCT of the spine showing bony spur at three levels. (f) Coronal section NCCT spine showing bony spur-DV6, DV10, DV12 (arrows). 
although the clinical-radiologic findings in this patient seem to substantiate the multiple accessory neuroenteric canal theory in the development of this unique type of SCM.

\section{Conflict of Interest}

None.

\section{Acknowledgments}

Department of Pediatrics, Army Hospital (Research \& Referral), New Delhi, India.

\section{References}

1 BremerJL. Dorsal intestinal fistula; accessory neurenteric canal; diastematomyelia. AMA Arch Pathol 1952;54(2):132-138

2 Pang D, Dias MS, Ahab-Barmada M. Split cord malformation: Part I: a unified theory of embryogenesis for double spinal cord malformations. Neurosurgery 1992;31(3):451-480

3 Pang D. Split cord malformation: Part II: clinical syndrome. Neurosurgery 1992;31(3):481-500

4 Khandelwal A, Tandon V, Mahapatra AK. An unusual case of 4 level spinal dysraphism: multiple composite type 1 and type 2 split cord malformation, dorsal myelocystocele and hydrocephalous. J Pediatr Neurosci 2011;6(1):58-61

5 Borkar SA, Mahapatra AK. Split cord malformations: a two years experience at AIIMS. Asian J Neurosurg 2012;7(2):56-60 